\title{
MACHINING OF BIOCOMPATIBLE CERAMICS WITH FEMTOSECOND LASER PULSES
}

\author{
S. Fiedler ${ }^{1}$, R. Irsig ${ }^{1}$, J. Tiggesbäumker ${ }^{1}$, C. Schuster ${ }^{1}$, C. Merschjann ${ }^{1}$, N. Rothe ${ }^{1}$, S. Lochbrunner ${ }^{1}$, M. Vehse ${ }^{2}$, H. \\ Seitz ${ }^{2}$, E.-D. Klinkenberg ${ }^{3}$ and K.-H. Meiwes-Broer ${ }^{1}$ \\ ${ }^{1}$ Institute of Physics, University of Rostock, Germany \\ ${ }^{2}$ Chair of Fluid Technology and Microfluidics, University of Rostock, Germany \\ ${ }^{3}$ DOT GmbH, Rostock, Germany \\ steffen.fiedler@uni-rostock.de
}

\begin{abstract}
Alumina toughened zirconia (ATZ) ceramic composites have been machined and structured with ultrashort laser pulses. With this approach the fabrication of various cavity patterns on the surface of the material with high precision becomes feasible. The scope of surface manipulation with femtosecond lasers gives opportunity to adopt the ceramic surface to tissue apposition for several different medical applications employing biocompatible ceramics.
\end{abstract}

Keywords: laser machining, biocompatible ceramics, ultrashort laser pulses

\section{Introduction}

Laser applications involving ceramics are versatile, covering among others laser deposition and ablation processes. For example, pulsed laser deposition has been successfully exploited as the premier research tool to produce high quality thin films of selected complex oxide materials [1]. Furthermore, micro-electronic substrates are produced out of alumina ceramics by laser cutting since ultrashort laser pulses have been proven as an excellent precision tool in micro-machining [2].

Alumina toughened zirconia (ATZ) is attractive in medical bearing applications, e.g. joint replacements, because of its low wear and its persistence. On the other hand its surface is inert and if strong tissue contact is required it can be only realized by surface structuring so that the tissue can find as much as possible contact areas.

A serious problem is the degradation of the mechanical properties of the ceramics by classical abrasive surface structuring methods with particle momentum transfer, which leads to unwanted side effects like e.g. enhanced crack propagation.

Here laser machining comes into play. Whereas for the marking of glass the formation of micro-cracks by laser radiation is essential, in contrast especially ultrashort pulse lasers promise a gentle surface treatment. In this study we have prepared and investigated several surface patterns on ATZ possibly suitable for tissue apposition.

\section{Methods}

Intense ultrashort laser pulses are applied to the mechanically polished surface of $2 \mathrm{~mm}$ thick discs of ATZ ceramics (diameter $30 \mathrm{~mm}$ ). For the studies described in this paper a laser setup that delivers pulses with a length as short as 100 to $150 \mathrm{fs}$ at a center wavelength of $793 \mathrm{~nm}$ and a pulse repetition rate of $1 \mathrm{kHz}$ is used. The experiment is equipped with a 3 -axis piezoelectric scanner and a lens with $100 \mathrm{~mm}$ focal length so that the laser spot position on the sample can be controlled with a resolution of about $10 \mu \mathrm{m}$ and micrometer precision.

\section{Results and Discussion}

Figure 1 shows a cutting line across a ceramic surface intersected by single holes drilled into the sample by 1000 laser pulses with an energy of $2 \mathrm{~mJ}$ each.

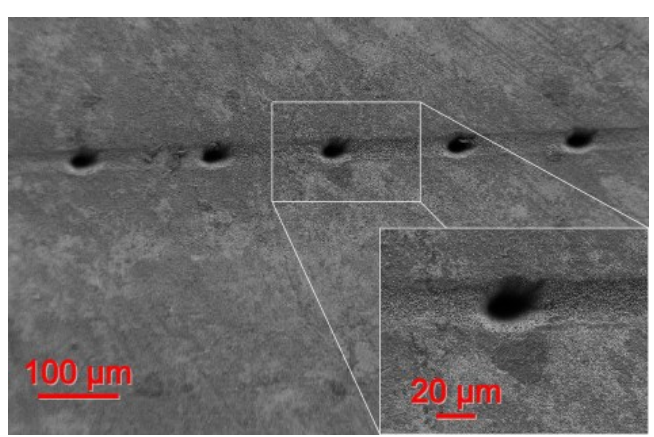

Figure 1: Narrow cutting line combined with a linear hole pattern drilled by femtosecond laser pulses in ATZ ceramics

The size of the written patterns is close to the diffraction limit. Despite the small dimensions a very precise and sharp-edged ablation can be realized. By changing the lasers focus conditions it is possible to create large coneshaped cavities. Many such cavities arranged adjacent in a rectangular 2D-pattern are shown in Figure 2.

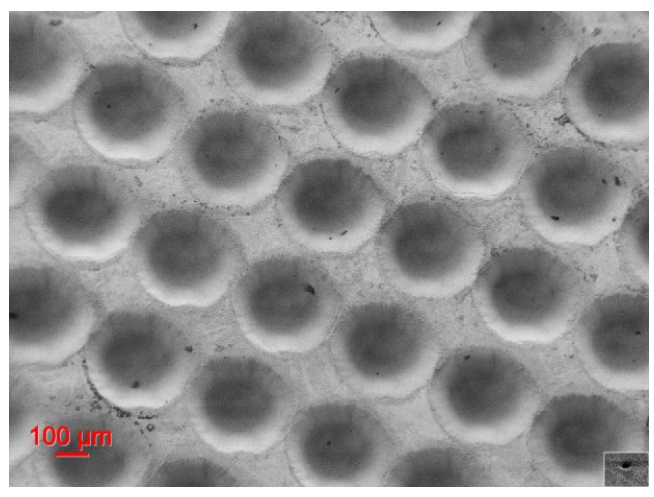

Figure 2: Rectangular cavity pattern drilled by defocused femtosecond laser pulses compared to small-scale machining from Figure 1 
The small inset at the lower right gives a size comparison to the machining results with tightly focused radiation and displays the magnified part of Figure 1. With such a patterning the total surface area is increased by a factor of three assuming cone-shaped cavities with an aspect ratio of one which can easily be accomplished. Thinking of drug-eluting applications these depots would be capable to comprise a much larger quantity of medical drugs as compared to the exemplary drug depots discussed in Ref. [3].

The unmachined surface surrounding the holes does not reveal any evidence of material degradation such as micro-cracks as discussed above which is demonstrated in Figure 3. Similar results were also found for the ablation of glass which is comparable with respect to the material class (see inset of Figure 3).

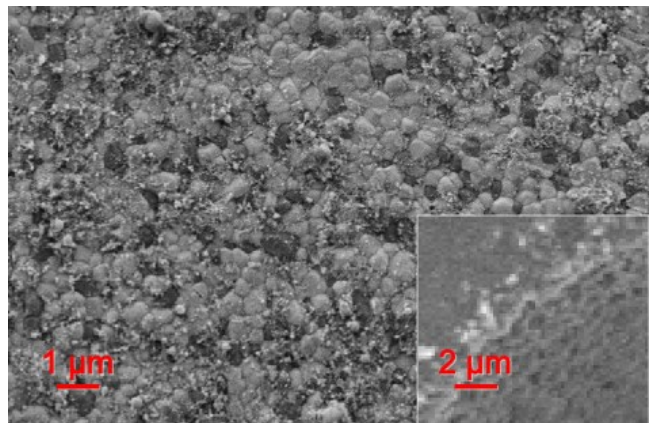

Figure 3: High magnification REM-image of the untouched ceramic surface around the holes; the inset shows a part of a hole in glass surface and its adjacent area

As shown in Figure 4 one can build up macroscopic surface patterns from closely spaced, deeply drilled holes as well. The spacing of the large holes is close enough to form paraboloid-like structures that are known to provide a high mechanical stability [4]. The inset gives information about the three-dimensional geometry of the structures. It features a contour plot with the highest elevations colored in red and areas lying furthest below the surface in dark blue. Additionally, depth profiles along the dotted lines are shown for the horizontal direction above and for the vertical direction to the right of the contour plot respectively.

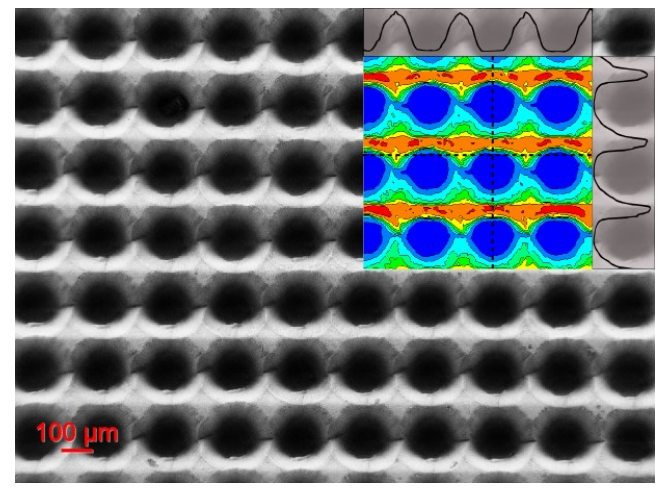

Figure 4: Formation of a regular surface pattern by deep hole drilling in ATZ ceramics

\section{Conclusions and Outlook}

Material ablation of ATZ ceramics by femtosecond laser pulses provides promising advantages in comparison with classical abrasive surface structuring methods. It offers precise machining with small influence on the materials integrity. Quality of the machining process is maintained throughout different sizes of the written patterns. By altering the effective surface area cell adhesion can be controlled [5].

Furthermore, the influence of varying laser pulse parameters such as pulse duration and temporal shape should be examined for machining of ceramics similar to the studies performed on polymers and glass (see Ref. [6] and [7]).

However, further investigations still have to verify the conservation of the outstanding mechanical properties of the ceramics after surface structuring by ultrashort pulse lasers.

\section{Acknowledgement}

The Federal Ministry of Education and Research (BMBF) supports REMEDIS as part of its "Leading-Edge Research and Innovation in the New German Länder" program (project number FKZ: 03IS2081) with the goal of a "Higher quality of life through novel micro-implants".

Furthermore we would like to thank the "Institute of Electronic Appliances and Circuits" from the "Faculty of Computer Science and Electrical Engineering" for taking the SEM pictures of the samples.

\section{Bibliography}

[1] Handbook of laser technology and applications / ed. by Colin E. Webb, Vol. 3: Applications, Bristol [u.a.] : IoP, Inst. of Physics Publ., 2004.

[2] C. Momma, S. Nolte, B. N. Chichkov, F. v. Alvensleben and A. Tünnermann. Appl. Surf. Science, vol. 109-110, pp. 15, 1997.

[3] M. Vehse, M. Gieseke, S. Fiedler, S. Petersen, R. Irsig, V. Senz, M. Löbler, M. Hustedt, S. Kaierle, H. Haferkamp, K. Sternberg, K.-P. Schmitz, S. Lochbrunner, K.H. Meiwes-Broer, H. Seitz. Biomed. Tech., vol. 57 (suppl. 1), pp. 1089, 2012.

[4] Chan, Esmond C., and Alex C. Scordelis. Shell and spatial structures engineering: proceedings of the International Symposium on Shell and Spatial Structures held at COPPE, Federal University of Rio de Janeiro, Brazil, 1983. Pentech Press, 1984.

[5] M. Loebler, M. Vehse, H. Seitz, and K. P. Schmitz. Biomed. Tech., vol. 57 (suppl. 1), pp. 423, 2012.

[6] C. Schuster, N. Rothe, A. V. Svanidze, S. Fiedler, R. Irsig, J. Tiggesbäumker, V. Senz, M. Vehse, H. Seitz, S. Lochbrunner and K.-H. Meiwes-Broer. Biomed. Tech., vol. 57 (suppl. 1), pp. 894, 2012.

[7] L. Englert, M. Wollenhaupt, L. Haag, C. SarpeTudoran, B. Rethfeld, T. Baumert, Appl. Phys. A, vol. 92, pp. 749, 2008. 